\title{
Risk for Nonalcoholic Fatty Liver Disease in Young Adults Born Preterm
}

\author{
Laura M. Breij Gerthe F. Kerkhof Anita C.S. Hokken-Koelega \\ Subdivision of Endocrinology, Department of Pediatrics, Erasmus Medical Centre/Sophia Children's Hospital, \\ Rotterdam, The Netherlands
}

\section{Key Words}

Nonalcoholic fatty liver disease $\cdot$ Weight gain · Infant growth · Catch-up

\begin{abstract}
Background: Nonalcoholic fatty liver disease (NAFLD) is considered the hepatic manifestation of metabolic syndrome. Accelerated catch-up in weight during infancy in subjects born at full term has been associated with increased risk for NAFLD in adulthood, but this association has not been studied in subjects born preterm. Methods: In 162 young adults born at a gestational age $<36$ weeks, we assessed the associations between fatty liver index (FLI, 0-100) and birth weight standard deviation score and first-year weight gain. We performed comparisons between subjects with and without accelerated catch-up in weight in the first year after term age. An FLI score was assigned to each participant to determine the clinical relevance, and regression analyses were performed. Results: Accelerated weight gain in the first 3 months after term age was associated with FLI as a continuous variable, whereas gestational age and low birth weight were not. Of the subjects with accelerated catch-up in weightfor-length after term age, $7.3 \%$ had a high FLI at the age of 21 years, whereas none of the subjects without accelerated catch-up in weight had a high FLI. Conclusion: Our study
\end{abstract}

shows that accelerated weight gain after term age is associated with an increased risk of developing NAFLD in young adults born preterm.

(c) 2015 S. Karger AG, Basel

\section{Introduction}

Nowadays, $8-10 \%$ of all newborns in developed countries are born preterm, and due to advances in neonatal intensive care, most of them reach adulthood [1]. Preterm infants are frequently exposed to fetal growth restriction, glucocorticoid treatment, and stressful events, which may contribute to the development of organ dysfunction and adverse vascular outcome later in life. In recent years, long-term outcomes for young adults born preterm were found to be less favorable than for those born at full term. Adults born preterm are at an increased risk of developing cardiovascular diseases [2-4] and have increased cardiovascular mortality $[5,6]$.

Nonalcoholic fatty liver disease (NAFLD) is considered to be the hepatic manifestation of metabolic syndrome and has been associated with several risk factors for cardiovascular diseases [7]. As NAFLD is strongly associated with obesity, its prevalence is rapidly increasing [8].

\section{KARGER 125\%}

(c) 2015 S. Karger AG, Base

$1663-2818 / 15 / 0843-0199 \$ 39.50 / 0$

E-Mail karger@karger.com

www.karger.com/hrp
Laura M. Breij, MD

Erasmus Medical Centre/Sophia Children's Hospital

Room No. Sk-0152, Dr. Molenwaterplein 40

NL-3015 GJ Rotterdam (The Netherlands)

E-Maill.breij@erasmusmc.nl 
Recently, our group showed that young adults who were born at full term and underwent accelerated weight gain during infancy had an increased fatty liver index (FLI), a measure of NAFLD in early adulthood [9]. While the gold standard for determining NAFLD is liver biopsy, the FLI is widely used and has recently been validated for identifying NAFLD in a large population-based study [10-14]. The FLI is a measure to predict hepatic steatosis in the general population, using an algorithm based on body mass index (BMI), waist circumference, triglycerides, and gamma-glutamyltransferase $(\gamma-\mathrm{GT})$, with, in case of a high FLI score $(>60)$, a positive predictive value of $72.2 \%$ in detecting fatty liver.

Because many preterm infants are prone for accelerated weight gain after an initial period of weight loss [15], we hypothesized that adults born preterm with accelerated weight gain after term age are at an increased risk for NAFLD compared with those without accelerated weight gain during infancy. We also hypothesized that adults born preterm have a higher FLI than adults born at full term $[9,16,17]$. We therefore investigated associations of birth weight, gain in weight-for-length, and accelerated catch-up in weight in the first year after term age with the FLI in general and with individual biochemical markers of FLI as well as with other markers indicating liver damage in a cohort of young adults born preterm $[7,18-21]$.

\section{Materials and Methods}

\section{Subjects}

The study population consisted of 162 healthy young adults (PREMS study) who were registered because of being born preterm (gestational age $<36$ weeks). The data of the participants were compared with the data of 268 young adults born at full term (PROGRAM study) [9]. Both cohorts had similar in- and exclusion criteria and measurements and were from the same research center, but they had a different gestational age. All included subjects were aged 18-24 years, Caucasian, born singleton, and had an uncomplicated neonatal period without severe asphyxia (defined as an Apgar score $<3$ after 5 minutes), sepsis, or long-term complications of respiratory ventilation and/or oxygen supply. Birth data were obtained from medical records, primary health care records, and general practitioner records. Data on educational level, socioeconomic status, use of medication, and alcohol use were obtained using questionnaires [22]. None of the participants had alcohol abuse, defined as a maladaptive pattern of alcohol use leading to clinically significant impairment or distress. Weight and length at $3,6,9$, and 12 months after birth had been measured prospectively at primary health care centers or hospitals. The Medical Ethics Committee of Erasmus Medical Centre, Rotterdam, The Netherlands, approved the study. Written informed consent was obtained from all participants.

\section{Measurements}

All participants visited the Erasmus Medical Centre in Rotterdam and were reimbursed for travel expenses. Prior to the visit, the participants had fasted for at least $12 \mathrm{~h}$. All anthropometric measurements were performed twice; the mean value was used for the analyses. Height was measured to the nearest $0.1 \mathrm{~cm}$ by a Harpenden stadiometer and weight to the nearest $0.1 \mathrm{~kg}$ by a Servo Balance KA-20-150S scale. Waist circumference was measured to the nearest $0.1 \mathrm{~cm}$ using a plastic flexible measuring tape. It was measured at the midway between the lower margin of the lowest rib and the upper margin of the iliac crest, at the end of a normal expiration.

All fasting blood samples were drawn between $08.00-13.00 \mathrm{~h}$, centrifuged after clotting, and kept frozen until assayed $\left(-80^{\circ} \mathrm{C}\right)$. All serum parameters were determined in the same laboratory. Triglycerides were measured using the GPO-PAP reagent kit (Roche Diagnostics). $\gamma$-GT, alkaline phosphatase, alanine aminotransferase, and aspartate aminotransferase levels were determined using standard laboratory routines.

\section{Statistical Analysis}

Standard deviation (SD) scores (SDS) for birth length, birth weight, and first year weight and length were calculated to correct for gestational age and sex [23]. SDS for adult height and weight were calculated to correct for sex and age [24]. The FLI was used to determine the risk for fatty liver disease [18]. The FLI is calcu-

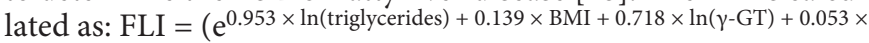

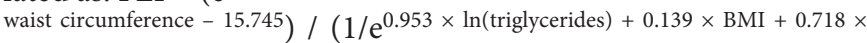
$\ln (\gamma-\mathrm{GT})+0.053 \times$ waist circumference -15.745$) \times 100$, and varies between 0 and 100. An FLI score was assigned to the participants as follows: an FLI $<30$ corresponds to a low risk (FLI score 1 , specificity $=$ $87 \%$ ), 30-60 to an intermediate risk (FLI-score 2), and $>60$ to a high risk (FLI score 3 , specificity $=86 \%$ for fatty liver) [18].

Multiple linear regression analyses were performed to investigate the association between weight gain per each 3 months during 1 year after term age, and several markers of NAFLD and liver damage in young adults born preterm. The 4 periods were analyzed separately from each other. In addition, we investigated the effect of weight gain from birth to term age on the FLI at 21 years. We performed a multiple regression analysis with the FLI at 21 years as dependent variable, as independent variables gestational age, gender, socioeconomic status, and adult age, and as dummy a decrease of weight SDS $\geq 1$ between birth and term age and no decrease in weight SDS.

Ordinal regression analyses were performed to determine the associations of FLI scores with birth weight SDS, birth length SDS, and weight gain during the 5 periods in the first year of life.

All regression analyses were adjusted for age, gender, gestational age, and socioeconomic status. To investigate the association between weight and outcome variables independently of length, as a measure for adiposity, adjustments were made for length growth during the same period.

Catch-up in weight in the first year after term age was defined as an increase in weight SDS of $>0.67$, because this represents the width of each percentile band on standard growth curves (2nd9th percentile, 9 th-25th percentile, etc.) [25].

Of the group with a catch-up in weight, one subgroup was formed based on accelerated $(S D \geq 0.5)$ weight gain during the first 3 months after term age. Additionally, young adults born preterm with accelerated catch-up and without accelerated catch- 
Table 1. Clinical characteristics of the total preterm group and subjects with accelerated versus nonaccelerated catch-up growth in weight in the first year after term age

\begin{tabular}{|c|c|c|c|c|c|c|c|c|c|c|}
\hline & \multicolumn{4}{|l|}{ Preterm } & \multicolumn{4}{|l|}{ Term } & \multicolumn{2}{|c|}{ Preterm vs. term } \\
\hline Male, \% & 50 & 41.8 & 50 & 0.443 & 107 & 31.5 & 57.9 & 0.042 & 0.267 & 0.585 \\
\hline Age, years & $20.8(1.68)$ & $20.5(1.81)$ & $20.8(1.76)$ & 0.595 & 20.9 (1.66) & 20.7 (1.68) & $21.0(1.94)$ & 0.557 & 0.683 & 0.688 \\
\hline Birth & $-0.51(1.80)$ & $-0.88(1.75)$ & $-1.16(1.56)$ & 0.439 & $-1.12(1.36)$ & $-2.19(0.69)$ & $-2.09(0.90)$ & 0.620 & $<0.001$ & 0.007 \\
\hline Term age & $-1.42(1.17)$ & $-1.58(1.18)$ & $-1.99(0.98)$ & 0.088 & - & - & - & - & - & - \\
\hline 3 months post-term & $-1.01(1.38)$ & $-0.34(1.38)$ & $-1.99(1.07)$ & $<0.001$ & $-0.91(1.10)$ & $-0.70(0.87)$ & $-2.13(0.95)$ & $<0.001$ & 0.114 & 0.625 \\
\hline 6 months post-term & $-0.77(1.23)$ & $-0.06(1.16)$ & $-1.28(0.94)$ & $<0.001$ & $-0.83(1.02)$ & $-0.40(0.80)$ & $-1.51(0.81)$ & $<0.001$ & 0.072 & 0.388 \\
\hline 9 months post-term & $-0.68(1.14)$ & $0.01(1.10)$ & $-0.91(0.93)$ & $<0.001$ & $-0.80(1.07)$ & $-0.36(0.73)$ & $-1.00(0.82)$ & 0.002 & 0.045 & 0.740 \\
\hline \multicolumn{11}{|l|}{ Length SDS } \\
\hline 3 months post-term & $-1.30(1.22)$ & $-0.98(1.18)$ & $-1.95(1.11)$ & $<0.001$ & $-1.02(1.21)$ & $-1.04(1.01)$ & $-1.71(1.14)$ & 0.018 & 0.787 & 0.464 \\
\hline 6 months post-term & $-0.98(1.09)$ & $-0.56(0.97)$ & $-1.45(0.88)$ & $<0.001$ & $-0.94(1.19)$ & $-0.61(0.99)$ & $-1.60(1.08)$ & $<0.001$ & 0.789 & 0.568 \\
\hline 9 months post-term & $-0.68(1.14)$ & $-0.18(0.88)$ & $-0.94(0.80)$ & $<0.001$ & $-0.91(1.26)$ & $-0.45(0.93)$ & $-1.27(1.03)$ & 0.002 & 0.122 & 0.190 \\
\hline 12 months post-term & $-0.66(1.17)$ & $0.02(0.91)$ & $-0.72(0.81)$ & $<0.001$ & $-0.92(1.20)$ & $-0.42(0.94)$ & $-1.16(1.02)$ & 0.005 & 0.015 & 0.084 \\
\hline Adult height SDS & $-0.42(0.95)$ & $-0.18(0.97)$ & $-0.30(0.91)$ & 0.553 & $-1.00(1.36)$ & $-0.63(0.91)$ & $-1.01(0.91)$ & 0.118 & 0.015 & 0.008 \\
\hline Adult weight SDS & $-0.28(1.22)$ & $0.19(1.09)$ & $-0.57(1.02)$ & 0.001 & $-0.63(1.43)$ & $0.04(1.12)$ & $-0.72(1.03)$ & 0.011 & 0.469 & 0.618 \\
\hline
\end{tabular}

Data are given as mean (SD). Accelerated catch-up $=$ increase in weight SDS $>0.67$ in the first year of life and $>0.5$ in the first 3 months; nonaccelerated catch-up $=$ increase in weight SDS $>0.67$ in the first year of life and $<0.5$ in the first 3 months. ${ }^{*}$ Accelerated versus nonaccelerated. Significant $p$ values are indicated in bold.

up in weight were compared to those born at full term. Differences in clinical characteristics were determined by an independent $t$ test.

Statistical package SPSS version 20.0 (SPSS, Inc., Chicago, Ill., USA) was used for analyses. Results were regarded statistically significant if $\mathrm{p}$ was $<0.05$ (two-sided).

\section{Results}

Clinical characteristics, the FLI and its components, and other liver markers are shown in tables 1 and 2. The mean (SD) FLI was 12.7 (16.4). A high FLI score was present in $5 \%$ of the total preterm study group.

\section{Fatty Liver Index}

Total Study Population of Adults Born Preterm

In table 3 , the results from the linear regression analyses are shown, with the FLI (0-100) and individual components of the FLI (triglycerides, BMI, waist circumfer- ence, and serum levels of $\gamma$-GT) as dependent variables. The increase in weight SDS adjusted for length SDS in the first 3 months after term age was significantly associated with FLI at the age of 21 years (FLI increased with $29.1 \%$ per 1 SDS increase in weight gain, $\mathrm{p}=0.034$, model 2 ). In addition, the increase in weight SDS adjusted for length SDS in the first 3 months after term age showed a significant association with waist circumference $(p=0.004)$. Birth weight SDS and gestational age were not significantly associated with the FLI or with the individual components of the FLI (model 1).

In addition, subjects with a decrease in weight SDS $\geq 1$ between birth and term age had a higher FLI at the age of 21 years than those without any decrease in weight SDS $(\mathrm{p}=0.01)$.

\section{Adults Born Preterm with Catch-Up in Weight after Term Age}

To determine if the speed of weight gain after term age was associated with determinants of NAFLD in young 
Table 2. Determinants of NAFLD of the preterm and term groups, divided into subjects with accelerated and nonaccelerated catch-up growth in weight in the first year after term age

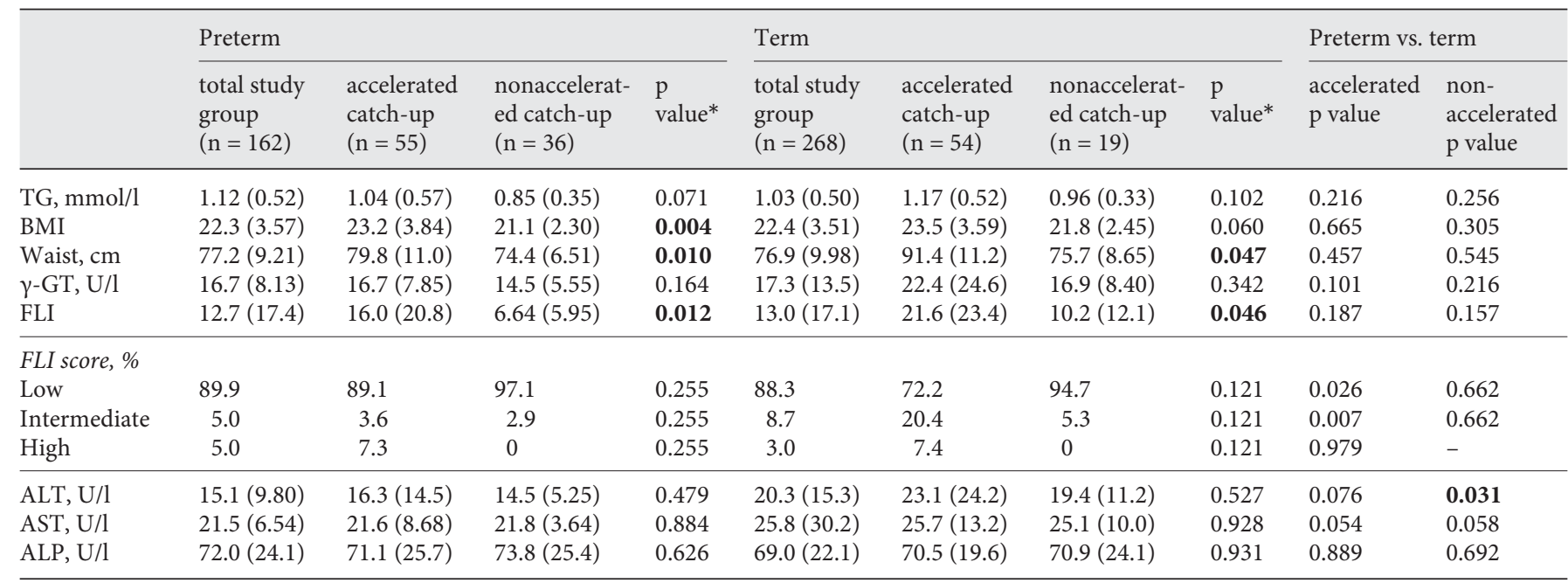

Data are given as mean $(\mathrm{SD})$. Waist $=$ waist circumference; $\mathrm{TG}=$ triglycerides; ALT= alanine aminotransferase; AST: aspartate aminotransferase; ALP $=$ alkaline phosphatase. An FLI score $<30$ corresponds to a low risk (FLI score 1), 30-60 to an intermediate risk (FLI score 2), and $>60$ to a high risk (FLI score 3) for fatty liver. Accelerated catch-up = increase in weight SDS $>0.67$ in the first year of life and $>0.5$ in the first 3 months; nonaccelerated catchup $=$ increase in weight SDS $>0.67$ in the first year of life and $<0.5$ in the first 3 months. * Accelerated versus nonaccelerated. Significant $p$ values are indicated in bold.

Table 3. Associations of birth size and early weight gain with the FLI and the components of the FLI at 21 years of age in young adults born preterm

\begin{tabular}{|c|c|c|c|c|c|c|c|c|c|c|c|c|c|c|c|c|}
\hline & & \multirow{2}{*}{\multicolumn{3}{|c|}{ FLI }} & \multicolumn{12}{|c|}{ FLI components } \\
\hline & & & & & \multicolumn{3}{|c|}{ triglycerides $^{\mathrm{a}}$} & \multicolumn{3}{|c|}{$\mathrm{BMI}^{\mathrm{a}}$} & \multicolumn{3}{|c|}{ waist $^{\mathrm{a}}$} & \multicolumn{3}{|c|}{$\gamma-\mathrm{GT}^{\mathrm{a}}$} \\
\hline & & $\beta$ & $\mathrm{p}$ & $\begin{array}{l}\text { adj. } \\
\mathrm{R}^{2}\end{array}$ & $\beta$ & $\mathrm{p}$ & $\begin{array}{l}\text { adj. } \\
\mathrm{R}^{2}\end{array}$ & $\beta$ & $\mathrm{p}$ & $\begin{array}{l}\text { adj. } \\
\mathrm{R}^{2}\end{array}$ & $\beta$ & $\mathrm{p}$ & $\begin{array}{l}\text { adj. } \\
\mathrm{R}^{2}\end{array}$ & $\beta$ & $\mathrm{p}$ & adj. $R^{2}$ \\
\hline Model $1^{\mathrm{b}}$ & Birth weight SDS & -6.32 & 0.446 & 0.042 & 0.10 & 0.989 & -0.044 & -2.12 & 0.107 & 0.040 & -1.55 & 0.090 & 0.140 & 5.87 & 0.103 & 0.054 \\
\hline Model $2^{c}$ & $\begin{array}{l}\text { Increase in weight SDS during } \\
3 \text { months after term age }\end{array}$ & 29.1 & 0.034 & 0.054 & 4.92 & 0.388 & -0.026 & 3.54 & 0.055 & 0.046 & 3.63 & 0.004 & 0.165 & 3.73 & 0.447 & 0.044 \\
\hline
\end{tabular}

${ }^{a} \log$ transformed (natural logarithm) for regression analyses. $\beta=$ Regression coefficient in \% ${ }^{b}$ Adjusted for gender, age, socioeconomic status, gestational age and birth length SDS. ${ }^{c}$ Adjusted for gender, age, socioeconomic status, gestational age and increase in length during 3 months after term age. Significant $\mathrm{p}$ values are indicated in bold.

adults born preterm, those with a catch-up in weight in the first year after term age were divided into 2 subgroups, either with or without accelerated catch-up in weight. Of all young adults with a clinically relevant catch-up in weight of at least 0.67 SDS in the first year after term age $(\mathrm{n}=91), 55 \mathrm{had}$ an increase in weight SDS $>0.5$ in the first 3 months after term age (accelerated catch-up), while 36 had no accelerated catch-up, defined as an increase in weight SDS $<0.5$ in the first 3 months after term age. The clinical characteristics of these 2 subgroups are shown in table 1.
Gestational age, sex, age, birth length SDS, and birth weight SDS were not different between the accelerated and nonaccelerated catch-up group. The accelerated catch-up group had a higher FLI $(\mathrm{p}=0.012)$, adult weight SDS ( $\mathrm{p}=0.001)$, BMI $(\mathrm{p}=0.004)$, and waist circumference $(p=0.010)$ at the age of 21 years than the nonaccelerated catch-up group, even after adjustment for gender, socioeconomic status, gestational age, and increase in length during the first year of life ( $\mathrm{p}=0.003$; fig. 1$)$.

To investigate whether the effect of accelerated catchup in weight in subjects born preterm was similar to that 


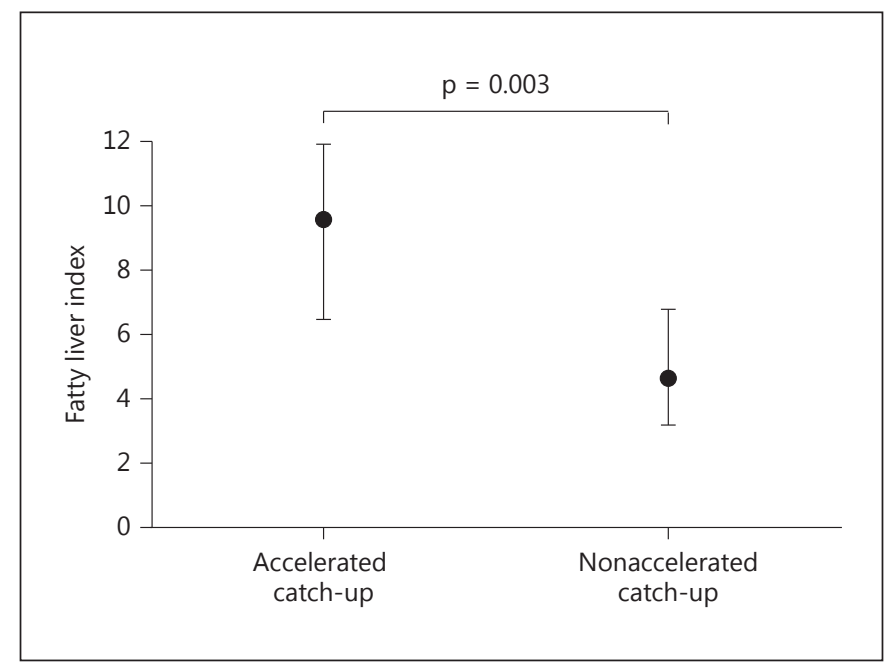

Fig. 1. Adjusted FLI of subjects with accelerated compared to those with nonaccelerated catch-up after term age. Estimated marginal means (95\% confidence intervals) are shown. Accelerated catchup $=$ increase in weight SDS $>0.67$ in the first year after term age and $>0.5$ in the first 3 months after term age. Nonaccelerated catch-up $=$ increase in weight SDS $>0.67$ in the first year after term age and $<0.5$ in the first 3 months after term age. Adjusted for gender, socioeconomic status, gestational age, and increase in length in the first year of life after term age.

in subjects born at full term, we compared the tempo of catch-up in young adults born preterm to those born at full term (table 2). Our data showed no difference between subjects born at full term and those born preterm.

\section{Fatty Liver Index Score}

An FLI score was assigned to each participant in order to obtain an NAFLD risk score (1: low, 2: intermediate, or 3: high). Of all adults born preterm, $5 \%(n=8)$ had a high FLI score, and $5 \%(n=8)$ had an intermediate FLI score (table 2). In the accelerated catch-up group, $7.3 \%$ had a high FLI score, and 3.6\% had an intermediate FLI score, while in the nonaccelerated catch-up group, $0 \%$ had a high FLI score, and $2.9 \%$ had an intermediate score. Lower birth weight and gestational age were not associated with a higher FLI score at 21 years of age.

\section{Discussion}

Our study in 162 young adults born preterm shows that accelerated infant weight gain after term age is associated with an increased risk of developing NAFLD in early adulthood. We also demonstrate that subjects born preterm with accelerated weight gain after term age have a significantly higher FLI in early adulthood than subjects without accelerated catch-up in weight, independent of size at birth and gestational age. Those findings are similar to those found in young adults born at full term.

We found an association between accelerated weight gain during infancy in preterm subjects and a higher NAFLD risk in early adulthood. Previous studies reported associations between small body size at birth and risk factors for NAFLD in adulthood [26, 27], but associations with preterm birth were not investigated. Some studies showed an association between preterm birth and waist circumference, a component of the FLI, but none of these studies investigated any associations with the FLI $[28,29]$.

Since our study population consisted of young adults, hard endpoints such as NAFLD and steatohepatitis could not be studied. The FLI was used as a proxy for a clustering of risk factors, a condition which precedes hepatic steatosis. A high FLI score $(>60)$ has a predictive value of $72.2 \%$ for a fatty liver [18], and although our study population was relatively young and healthy, with a relatively low BMI, a high FLI score was found in $5 \%$ of the subjects born preterm.

In addition, it was striking that $10.9 \%$ of the subjects born preterm with accelerated catch-up in weight-forlength after term age had a high or intermediate FLI at the age of 21 years, compared with only $2.9 \%$ of the subjects without accelerated catch-up in weight-for-length. We previously showed a similar association between accelerated infant weight gain and increased risk for NAFLD in young adults born at full term [9]. Our present study shows that this association is also apparent in young adults born preterm. Besides this, our study shows that preterm subjects with a decrease in weight SDS $\geq 1$ between birth and term age had a higher FLI at 21 years than those without any decrease in weight SDS. Both these findings indicate that a balance in neonatal increase in weight compared to length is also important for preterm infants to reduce the risk of developing NAFLD. This may be achieved by modifying nutritional intervention according to weight-for-length trajectories, particularly in the first months after term age. These data should not be interpreted to the effect that we recommend slow weight gain in preterm babies, but we discourage accelerated catch-up in weight-for-length after term age. They also point out that not only weight but also length should be routinely measured during infancy. Earlier studies showed that accelerated weight gain, mediated by nutrition-enriched diets, has adverse effects on cardiovascular risk factors in later life $[6,30]$. Our findings warrant that 
parents should be informed about the long-term effects of accelerated weight gain, as the first months after the delivery of preterm infants, usually up to around term age, seem to be a critical window for the programming of later body composition.

We acknowledge that when investigating differences between subjects born preterm and those born at full term, differences in early nutritional intake should be taken into account. The prevalence and duration of breastfeeding is lower in infants born preterm [31]. Formulafed infants have a higher risk of being overweight in childhood and later in life $[32,33]$. Infant feeding might thus influence a later risk for NAFLD. Studies in rats showed that the liver might act as a systemic buffer, largely increasing its lipid content in the early stage of high-fat feeding [34]. It has been suggested that breastfeeding protects against the development of nonalcoholic steatohepatitis (NASH) in children [35]. We did not have sufficient nutritional data to investigate differences in nutritional intake in early life between adults born preterm and those born at full term and its relation with growth in infancy and the risk for NAFLD, but our results warrant further investigations.

In conclusion, our study shows that accelerated increase in weight compared to length in the first 3 months after term age in subjects born preterm is associated with a higher risk of developing NAFLD in adulthood. Longterm prospective studies are warranted to study the effects of various infant feeding practices on the risk for NAFLD.

\section{Acknowledgements}

We greatly thank Mrs. J. Dunk, research nurse, for her technical assistance and support with the data collection.

\section{Disclosure Statement}

The authors have nothing to disclose.

\section{References}

1 Blencowe $\mathrm{H}$, et al: National, regional, and worldwide estimates of preterm birth rates in the year 2010 with time trends since 1990 for selected countries: a systematic analysis and implications. Lancet 2012;379:2162-2172.

2 Thomas EL, et al: Aberrant adiposity and ectopic lipid deposition characterize the adult phenotype of the preterm infant. Pediatr Res 2011;70:507-512.

-3 Evensen KA, et al: Effects of preterm birth and fetal growth retardation on cardiovascular risk factors in young adulthood. Early Hum Dev 2009;85:239-245.

4 Kerkhof GF, et al: Does preterm birth influence cardiovascular risk in early adulthood? J Pediatr 2012;161:390-396.e1.

5 Crump C, et al: Gestational age at birth and mortality in young adulthood. JAMA 2011; 306:1233-1240.

6 Singhal A, et al: Low nutrient intake and early growth for later insulin resistance in adolescents born preterm. Lancet 2003;361:10891097.

7 Nguyen QM, et al: Elevated liver function enzymes are related to the development of prediabetes and type 2 diabetes in younger adults: the Bogalusa Heart Study. Diabetes Care 2011;34:2603-2607.

8 Vernon G, Baranova A, Younossi ZM: Systematic review: the epidemiology and natural history of non-alcoholic fatty liver disease and non-alcoholic steatohepatitis in adults. Aliment Pharmacol Ther 2011;34:274-285.
-9 Breij LM, Kerkhof GF, Hokken-Koelega AC: Accelerated infant weight gain and risk for nonalcoholic fatty liver disease in early adulthood. J Clin Endocrinol Metab 2014;99:1189_ 1195.

10 Koehler EM, et al: External validation of the fatty liver index for identifying nonalcoholic fatty liver disease in a population-based study. Clin Gastroenterol Hepatol 2013;11:12011204.

11 Grattagliano I, et al: Utility of noninvasive methods for the characterization of nonalcoholic liver steatosis in the family practice. The 'VARES' Italian multicenter study. Ann Hepatol 2013;12:70-77.

12 Barchetta I, et al: Strong association between non alcoholic fatty liver disease (NAFLD) and low $25(\mathrm{OH})$ vitamin $\mathrm{D}$ levels in an adult population with normal serum liver enzymes. BMC Med 2011;9:85

-13 de Andrade AR, et al: Nonalcoholic fatty liver disease in severely obese individuals: the influence of bariatric surgery. Ann Hepatol 2008;7:364-368.

14 Gastaldelli A, et al: Fatty liver is associated with insulin resistance, risk of coronary heart disease, and early atherosclerosis in a large European population. Hepatology 2009;49: 1537-1544.

15 Moyses HE, et al: Early parenteral nutrition and growth outcomes in preterm infants: a systematic review and meta-analysis. Am J Clin Nutr 2013;97:816-826.
16 Kerkhof GF, Hokken-Koelega AC: Rate of neonatal weight gain and effects on adult metabolic health. Nat Rev Endocrinol 2012;8: 689-692.

17 Leunissen RW, et al: Timing and tempo of first-year rapid growth in relation to cardiovascular and metabolic risk profile in early adulthood. JAMA 2009;301:2234-2242.

18 Bedogni G, et al: The Fatty Liver Index: a simple and accurate predictor of hepatic steatosis in the general population. BMC Gastroenterol 2006;6:33

19 Fraser A, et al: The associations between birthweight and adult markers of liver damage and function. Paediatr Perinat Epidemiol 2008;22:12-21.

20 Miyake T, et al: Metabolic markers and ALT cutoff level for diagnosing nonalcoholic fatty liver disease: a community-based cross-sectional study. J Gastroenterol 2012;47:696703.

21 Kocabay G, et al: Alkaline phosphatase: can it be considered as an indicator of liver fibrosis in non-alcoholic steatohepatitis with type 2 diabetes? Bratisl Lek Listy 2011;112:626-629.

22 Statistics Netherlands: Dutch Standard Classification of Education, 2006. Available from www.cbs.nl/en-GB.

23 Usher R, McLean F: Intrauterine growth of live-born Caucasian infants at sea level: standards obtained from measurements in 7 dimensions of infants born between 25 and 44 weeks of gestation. J Pediatr 1969;74:901-910. 
24 Fredriks AM, et al: Continuing positive secular growth change in The Netherlands 19551997. Pediatr Res 2000;47:316-323.

25 Ong KK, et al: Association between postnatal catch-up growth and obesity in childhood: prospective cohort study. BMJ 2000;320:967971.

26 Sandboge S, et al: Early growth and non-alcoholic fatty liver disease in adulthood - the NAFLD liver fat score and equation applied on the Helsinki Birth Cohort Study. Ann Med 2013;45:430-437.

27 Faienza MF, et al: Nonalcoholic fatty liver disease in prepubertal children born small for gestational age: influence of rapid weight catch-up growth. Horm Res Paediatr 2013;79: 103-109.
28 Kerkhof GF, et al: Health profile of young adults born preterm: negative effects of rapid weight gain in early life. J Clin Endocrinol Metab 2012;97:4498-4506.

29 Finken MJ, et al: Lipid profile and carotid intima-media thickness in a prospective cohort of very preterm subjects at age 19 years: effects of early growth and current body composition. Pediatr Res 2006;59(4 Pt 1):604-609.

30 Singhal A, et al: Nutrition in infancy and longterm risk of obesity: evidence from 2 randomized controlled trials. Am J Clin Nutr 2010;92: 1133-1144.

1 Bertoncelli N, et al: Oral feeding competences of healthy preterm infants: a review. Int J Pediatr 2012;2012:896257.
32 Armstrong J, Reilly JJ, Child T: Health Information, Breastfeeding and lowering the risk of childhood obesity. Lancet 2002;359:20032004.

33 Gillman MW, et al: Risk of overweight among adolescents who were breastfed as infants. JAMA 2001;285:2461-2467.

34 Gauthier MS, Favier R, Lavoie JM: Time course of the development of non-alcoholic hepatic steatosis in response to high-fat dietinduced obesity in rats. Br J Nutr 2006;95: 273-281.

35 Nobili V, et al: A protective effect of breastfeeding on the progression of non-alcoholic fatty liver disease. Arch Dis Child 2009;94: 801-805. 